\title{
Cold gas in the Perseus cluster core: excitation of molecular gas in filaments
}

\author{
P. Salomé ${ }^{1, \star}$, F. Combes ${ }^{2}$, Y. Revaz ${ }^{2,6}$, A. C. Edge ${ }^{3}$, N. A. Hatch ${ }^{5}$, A. C. Fabian ${ }^{4}$, and R. M. Johnstone ${ }^{4}$ \\ ${ }^{1}$ Institut of Radio Astronomy (IRAM), Domaine Universitaire, 300 rue de la piscine, 38400 St Martin d'Hères, France \\ e-mail: salome@iram.fr \\ 2 Observatoire de Paris, LERMA, 61 Av. de l'Observatoire, 75014 Paris, France \\ 3 Department of Physics, University of Durham, South Road, Durham DH1 3LEi, UK \\ 4 Institute of Astronomy, Madingley Road, Cambridge CB3 OHA, UK \\ Leiden Observatory, 2300 RA Leiden, The Netherlands \\ ${ }^{6}$ Laboratoire d'Astrophysique, Ecole Polytechnique Fédérale de Lausanne (EPFL), Observatoire, 1290 Sauverny, Switzerland
}

Received 1 February 2008 / Accepted 13 March 2008

\section{ABSTRACT}

\begin{abstract}
We have recently detected CO lines in the well-known filaments around NGC 1275, the galaxy at the centre of the Perseus cluster of galaxies. These previous observations, with the HERA multi-beam array at the IRAM $30 \mathrm{~m}$ telescope enabled us to make a large map of the $\mathrm{CO}(2-1)$ line and to see hints of molecular gas far away from the cluster centre. To confirm the presence of $\mathrm{CO}$ emission lines in the outer filaments and to study the $\mathrm{CO}(2-1) / \mathrm{CO}(1-0)$ line ratio, we observed seven regions of interest again with the $30 \mathrm{~m}$ telescope in both $\mathrm{CO}(1-0)$ and $\mathrm{CO}(2-1)$. The regions we observed were: the eastern filament, the horseshoe, the northern filament and a southern extension, all selected from $\mathrm{H} \alpha$ emission line mapping. Molecular gas is detected in all the observed regions. This result confirms the large extent of the cold molecular gas filaments. We discuss the $\mathrm{CO}(2-1) / \mathrm{CO}(1-0)$ ratios in the filaments. The eastern filament has optically thick gas, whereas further away, the line ratio increases close to values expected for a warmer optically thin medium. We also show $\mathrm{CO}(1-0)$ and $\mathrm{CO}(2-1)$ lines in 9 regions closer to the centre. The kinematics of the $\mathrm{CO}$ is studied here in more detail and confirms that it follows the motions of the warm $\mathrm{H}_{2}$ gas found in the near-infrared. Finally, we searched for dense gas tracers around $3 \mathrm{C} 84$ and claim here the first detection of $\mathrm{HCN}(3-2)$.
\end{abstract}

Key words. galaxies: elliptical and lenticular, $\mathrm{cD}$ - galaxies: cooling flows - galaxies: intergalactic medium galaxies: individual: NGC 1275

\section{Introduction}

Over the past decade X-ray observations have shown a lack of cool X-ray emitting gas in cooling flow clusters (e.g. Peterson et al. 2003), and many feedback models have been proposed to explain the required energy injection into the intracluster medium (e.g. Binney \& Tabor 1995; Omma \& Binney 2004). At the same time, large amounts of molecular gas have been found in many Brightest Cluster Galaxies (BCG) (Edge 2001; Salomé \& Combes 2003), providing evidence that some of the ICM may cool to very low temperatures. Detailed studies of this cold molecular gas reservoir are a complementary way to probe feedback processes.

The giant cD galaxy NGC 1275 is the central galaxy of the Perseus cluster (Abell 426) and lies at a redshift of 0.01756. At this distance, $1^{\prime \prime}$ is $350 \mathrm{pc}\left(H_{0}=71 \mathrm{~km} \mathrm{~s}^{-1} \mathrm{Mpc}^{-1}, \Omega_{M}=0.27\right.$, $\left.\Omega_{\lambda}=0.73\right)$. This object is famous for the huge filamentary structure detected in the optical (Hu et al. 1983; Conselice et al. 2001). These bright $\mathrm{H} \alpha$ emitting filaments are known to be directly/indirectly associated with cooling cores (Crawford et al. 1999; Edwards et al. 2007). We have shown previously (Salomé et al. 2006, hereafter S06) that the molecular gas is also detected in $\mathrm{CO}(2-1)$ emission in the centre of NGC 1275 with morphology and dynamics identical to that of the $\mathrm{H} \alpha$ emission.

\footnotetext{
* Based on observations carried out at the IRAM 30 m telescope, with the help of IRAM members, Pico Veleta, Spain.
}

We present here the results of follow-up observations aimed at confirming the presence of cold gas associated with the optical filaments further out from the galaxy. We looked for $\mathrm{CO}(1-0)$ and $\mathrm{CO}(2-1)$ emission lines in 7 regions selected in the optical filaments. We also observed the central region at both 1.3 and $3 \mathrm{~mm}$ with high sensitivity receivers. Section 2 describes the observations. Section 3 presents the results and discusses the spectra obtained in the different regions of interest. We then compare the CO results (morphology, kinematics, line ratios) with data at other wavelengths in Sect. 4. Section 5 summarizes our conclusions.

\section{Observations}

The observations were made with the IRAM 30 m telescope on Pico Veleta, Spain during two different runs in 2006. We used the wobbler switching mode with two $3 \mathrm{~mm}$ and two $1.3 \mathrm{~mm}$ receivers operating simultaneously. The beam throw was 4 arcmin. Receivers were tuned to the $\mathrm{CO}(1-0)$ and $\mathrm{CO}(2-1)$ emission lines, redshifted to the velocity of NGC 1275 . Frequent pointings were done on $3 \mathrm{C} \mathrm{84}$, the radio source at the centre of NGC 1275. At $3 \mathrm{~mm}$, we used two $512 \times 1 \mathrm{MHz}$ filter-banks. This gives a total band of $\sim 1300 \mathrm{~km} \mathrm{~s}^{-1}$ for the $\mathrm{CO}(1-0)$ line setup. In addition, we used the two $250 \times 4 \mathrm{MHz}$ resolution filter-banks for the $1.3 \mathrm{~mm}$ receivers, providing a $1 \mathrm{GHz}$ band width, for the $1.3 \mathrm{~mm}$ receivers which also corresponds to a $1300 \mathrm{~km} \mathrm{~s}^{-1}$ bandwidth. 
The beams of the $30 \mathrm{~m}$ telescope, at $3 \mathrm{~mm}(113.282 \mathrm{GHz})$ and $1.3 \mathrm{~mm}(226.559 \mathrm{GHz})$, are respectively $22^{\prime \prime}$ and $11^{\prime \prime}$.

Regions close to the centre were very quickly detected (in 10-20 min), while the more distant regions required more time $(\sim 2 \mathrm{~h})$. The signals are expressed here in main beam brightness temperatures. The main-beam efficiency of the $30 \mathrm{~m}$ telescope is the ratio of the antenna temperature to the main beam temperature: $T_{\mathrm{A}}^{*} / T_{\mathrm{mb}}=B_{\text {eff }} / F_{\text {eff }}$ with the ratio of the beam efficiency to the forward efficiency being: $B_{\text {eff }} / F_{\text {eff }}=0.75 / 0.95$ at $3 \mathrm{~mm}$ and 0.52/0.91 at $1.3 \mathrm{~mm}$ (cf IRAM-30 m site http://www. iram. es/).

The data were calibrated with the MIRA software and reduced with the CLASS90 package. Spiky channels and bad scans were dropped and linear baselines were subtracted for each spectrum. After averaging all the spectra for each line at each position, the data were Hanning smoothed to a $42 \mathrm{~km} \mathrm{~s}^{-1}$ resolution.

The data are summarized in Tables 1 and 2. The $\mathrm{CO}(2-1)$ lines of the filaments use the present observations and the data obtained with HERA (S06): the $\mathrm{CO}(2-1)$ spectra were convolved with the beam pattern at $3 \mathrm{~mm}$ to compare the $\mathrm{CO}(1-0)$ and $\mathrm{CO}(2-1)$ temperatures.

\section{Results}

We detected $\mathrm{CO}$ in all the regions observed inside the $\mathrm{H} \alpha$ filaments. Offsets are relative to the $3 \mathrm{C} 84$ position: RA 03:19:48.15, Dec 41:30:42.1 (J2000). In the central region (indicated as centre 1 to 9 in Fig. 1 and Table 2), the $3 \mathrm{~mm}$ data suffered from baselines instabilities due to the strong continuum of $3 \mathrm{C} 84$. The $3 \mathrm{~mm}$ spectra shown on the left hand side of Fig. 4 are affected by those baseline ripples. The $1.3 \mathrm{~mm}$ data, not affected by baseline ripples give a more reliable idea of the line shape. Nevertheless $C O$ lines are detected in all regions except the region outside the H $\alpha$ filaments. This is the region named test, which has no $\mathrm{H} \alpha$ emitting counterpart. Our earlier HERA map (S06) showed a hint of a detection at this place, but this was not confirmed by the new observations.

In order to compute the molecular gas content from the integrated $\mathrm{CO}$ intensity $I_{\mathrm{CO}}\left(\mathrm{K} \mathrm{km} \mathrm{s}^{-1}\right)$, we used

$I_{\mathrm{CO}}=\int T_{\mathrm{mb}}(\mathrm{CO}) \mathrm{d} V$

where $T_{\mathrm{mb}}(\mathrm{CO})$ is the main beam antenna brightness temperature, obtained with the $\mathrm{CO}$ emission line and $\mathrm{d} V$ is the line width. We then adopted the standard Milky Way conversion factor commonly used for $N\left(\mathrm{H}_{2}\right)$ estimates:

$N\left(\mathrm{H}_{2}\right)=2.3 \times 10^{20} I_{\mathrm{CO}}\left(\right.$ molecules $\left./ \mathrm{cm}^{2}\right)$.

From this equation the mass of molecular hydrogen, contained in one beam is:

$M_{\mathrm{gas}}\left(M_{\odot}\right)=1.36 \times 2.95 \times 10^{-19} I_{\mathrm{CO}} \theta^{2} D^{2} \frac{N\left(\mathrm{H}_{2}\right)}{I_{\mathrm{CO}}}$,

$I_{\mathrm{CO}}$ is the integrated intensity in $\mathrm{K} \mathrm{km} \mathrm{s}^{-1}, \theta$ is the beamsize of the telescope in $\operatorname{arcsec}$, and $D$ is the distance of the galaxy, taken to be $72.6 \mathrm{Mpc}$. We also included a factor 1.36 to take into account the He contribution to the gas mass.

\subsection{The filaments}

We describe below the regions selected from our HERA map (see S06). These regions were chosen to be distant from the
East-West filament seen in $\mathrm{CO}(2-1)$ and known for their peculiar optical morphology. The first aim was to confirm the CO detections far away from the central galaxy. The regions called Off 1, Off 2 and Pos 2 trace the northern and southern filaments visible in $\mathrm{H} \alpha$ up to a projected distance of $25 \mathrm{kpc}$ north and south of the galaxy. Pos 11 is centred on a looped-back filament seen in optical emission lines that may trace uplifted gas behind a rising cold gas bubble inside the hot intracluster medium (Fabian et al. 2003; Hatch et al. 2006). Finally, closer to the centre, East and Off 3, are two fields in the eastern filament. The East region includes a young star cluster (Shields \& Filipenko 1990).

We now compare the velocity shift of our $\mathrm{CO}$ detections with the velocities of the warm $(2000 \mathrm{~K}) \mathrm{H}_{2}$ gas detected by Hatch et al. (2005) and the hot $\left(10^{4} \mathrm{~K}\right)$ optical line emitting gas measured by Hatch et al. (2006).

The eastern filament: refers to positions East and Off 3, at about $8 \mathrm{kpc}$ from the galaxy's centre. It corresponds to regions named A, B, C and D in Hatch et al. (2005). The CO emission lines are centred at $-100 \mathrm{~km} \mathrm{~s}^{-1}$ relative to the systemic velocity. This blueshift is the same as the velocity found for the $\mathrm{P} \alpha, \mathrm{Br} \gamma$ and $\mathrm{H}_{2} v=1-0 \mathrm{~S}(1), \mathrm{S}(2)$ and $\mathrm{S}(3)$ lines detected by the above mentioned authors.

The southern filament: refers to position Pos 2 at $25 \mathrm{kpc}$ from the galaxy's centre. It corresponds to regions named SW1 and SW2 in Hatch et al. (2005). Here again, the CO emission is blueshifted relative to the nucleus, like the infrared $\mathrm{H}_{2}$ tracers.

The horseshoe: refers to the position Pos 11, at least $25 \mathrm{kpc}$ from the galaxy's centre. The $\mathrm{CO}(1-0)$ line is detected at the limit of the sensitivity reached. There is also a tentative detection of $\mathrm{CO}(2-1)$ line which disappears when merged with the HERA data, so this detection is not very strong. Nevertheless, the two emission lines have positive velocities $\left(36 \mathrm{~km} \mathrm{~s}^{-1}\right.$ and $120 \mathrm{~km} \mathrm{~s}^{-1}$ ). This velocity range agrees with that from the infrared $\mathrm{H}_{2}$ tracers by Hatch et al. (2005). It is also the same velocity shift as the hot $\mathrm{H} \alpha$ emitting gas found by Hatch et al. (2006) in the loop of the horseshoe filament (see their Fig. 6).

The northern filament: This region refers to the position Off 2, at about $25 \mathrm{kpc}$ from the galaxy centre. The $\mathrm{H} \alpha$ emitting gas has a velocity range between +6 and $+41 \mathrm{~km} \mathrm{~s}^{-1}$ relative to the systematic velocity. This is similar to the velocity of the $\mathrm{CO}$ line.

The tangential filament: This region refers to position Off 1 , at least $12.3 \mathrm{kpc}$ from the galaxy centre. It corresponds to a region inside the tangential filament (slit 5) of Hatch et al. (2006). The CO velocity is not well defined here as it is not consistent between the $3 \mathrm{~mm}$ and the $1.3 \mathrm{~mm}$ data. However, there is a hint of a two-component velocity structure, at $\pm 100 \mathrm{~km} \mathrm{~s}^{-1}$. This is consistent with the $\mathrm{H} \alpha$ velocity found in the same region (distance between $\sim 15$ and $\sim 20 \mathrm{kpc}$ in Fig. 7 of Hatch et al. 2006).

The test region: A hint of a $\mathrm{CO}(2-1)$ detection was present in the S06 HERA data, in a region that corresponds to no $\mathrm{H} \alpha$ filament. We re-observed this region and found no $\mathrm{CO}(1-0)$ or $\mathrm{CO}(2-1)$ emission (i.e. $\left.M_{\text {gas }}<10^{8} M_{\odot}\right)$. We conclude that there is no, or very little, cold gas outside the $\mathrm{H} \alpha$ filaments.

\subsection{The central region}

We observed 9 regions to map the $\mathrm{CO}$ emission close to $3 \mathrm{C} 84$. We present here a fully sampled $\mathrm{CO}(1-0)$ map of about $50^{\prime \prime} \times 50^{\prime \prime}$ with a spatial resolution of $22^{\prime \prime}$. The $\mathrm{CO}(2-1)$ map does not fully sample the same region but has a better resolution of $11^{\prime \prime}$. All the results are described in more detail in Table 2 and Figs. 3 and 4. In the central regions the $\mathrm{CO}(1-0)$ and $\mathrm{CO}(2-1)$ line profiles are 
Table 1. Results of the observations.

\begin{tabular}{|c|c|c|c|c|c|c|c|c|}
\hline Position & $\begin{array}{c}\text { Offsets } \\
{\left[{ }^{\prime \prime} \times^{\prime \prime}\right]}\end{array}$ & Line & $\begin{array}{c}T_{\mathrm{mb}} \\
{[\mathrm{mK}]}\end{array}$ & $\begin{array}{l}\text { Velocity } \\
{\left[\mathrm{km} \mathrm{s}^{-1}\right]}\end{array}$ & $\begin{array}{c}\text { Width } \\
{\left[\mathrm{km} \mathrm{s}^{-1}\right]}\end{array}$ & $\begin{array}{c}I_{\mathrm{CO}} \\
{\left[\mathrm{K} \mathrm{km} \mathrm{s}^{-1}\right]}\end{array}$ & $\begin{array}{c}M_{\mathrm{gas}} \\
{\left[10^{8} M_{\odot}\right]}\end{array}$ & $\overline{T_{21} / T_{10}}$ \\
\hline Off1 & {$[3,33]$} & $\mathrm{CO}(1-0)$ & $2 \pm 1.0$ & $33.3 \pm 37.1$ & $237.6 \pm 59.8$ & $0.5 \pm 0.1$ & 1.1 & \\
\hline Off1 & {$[3,33]$} & $\mathrm{CO}(2-1)$ & $5.3 \pm 1.8$ & $-55.2 \pm 26$ & $275 \pm 47.7$ & $1.5 \pm 0.3$ & & \\
\hline Off1 & {$[3,33]$} & $\mathrm{CO}(2-1)$ & $4.7 \pm 0.8$ & $-18.4 \pm 12.9$ & $247.1 \pm 26.7$ & $1.2 \pm 0.1$ & & 2.4 \\
\hline Off2 & {$[3,69]$} & $\mathrm{CO}(1-0)$ & $2.5 \pm 0.6$ & $49 \pm 13.4$ & $128.5 \pm 31.1$ & $0.3 \pm 0.1$ & 0.8 & \\
\hline Off2 & {$[3,69]$} & $\mathrm{CO}(2-1)$ & $7.8 \pm 2.3$ & $-21.8 \pm 20.7$ & $210.6 \pm 43$ & $1.8 \pm 0.3$ & & \\
\hline Off2 & {$[3,69]$} & $\mathrm{CO}(2-1)$ & $4 \pm 1.2$ & $-29.3 \pm 25$ & $187.3 \pm 73.9$ & $0.8 \pm 0.2$ & & 1.6 \\
\hline Off3 & {$[21,-9]$} & $\mathrm{CO}(1-0)$ & $15.2 \pm 2.9$ & $-91.2 \pm 8.4$ & $93 \pm 16.9$ & $1.5 \pm 0.3$ & 3.5 & \\
\hline Off3 & {$[21,-9]$} & $\mathrm{CO}(2-1)$ & $9.4 \pm 3.1$ & $-88.1 \pm 24.7$ & $276.6 \pm 48.6$ & $2.77 \pm 0.5$ & & \\
\hline Off3 & {$[21,-9]$} & $\mathrm{CO}(2-1)$ & $8.2 \pm 0.9$ & $-62.8 \pm 6.5$ & $153.8 \pm 17.7$ & $1.34 \pm 0.1$ & & 0.5 \\
\hline Pos11 & {$[-45,51]$} & $\mathrm{CO}(1-0)$ & $1.5 \pm 0.7$ & $65 \pm 32.2$ & $272.6 \pm 119.9$ & $0.44 \pm 0.13$ & 1 & \\
\hline Pos 11 & {$[-45,51]$} & $\mathrm{CO}(2-1)$ & $3 \pm 1.5$ & $55 \pm 21.5$ & $96.2 \pm 45.6$ & $0.31 \pm 0.13$ & & $2^{*}$ \\
\hline Pos11 & {$[-45,51]$} & $\mathrm{CO}(2-1)$ & $\leq 3 \times 1.3$ & - & - & - & & \\
\hline Pos2 & {$[-45,-51]$} & $\mathrm{CO}(1-0)$ & $1.9 \pm 0.9$ & $-47.8 \pm 25.7$ & $116.9 \pm 48.1$ & $0.2 \pm 0.1$ & 0.5 & \\
\hline Pos2 & {$[-45,-51]$} & $\mathrm{CO}(2-1)$ & $3.8 \pm 1.6$ & $-65.4 \pm 27.9$ & $205 \pm 53.2$ & $0.8 \pm 0.2$ & & \\
\hline Pos2 & {$[-45,-51]$} & $\mathrm{CO}(2-1)$ & $2.3 \pm 1.0$ & $-64.4 \pm 23.2$ & $140.6 \pm 51.1$ & $0.3 \pm 0.1$ & & 1.2 \\
\hline East & {$[27,3]$} & $\mathrm{CO}(1-0)$ & $23.4 \pm 4.5$ & $-109.1 \pm 9.1$ & $106.2 \pm 20.1$ & $2.6 \pm 0.5$ & 6.2 & \\
\hline East & {$[27,3]$} & $\mathrm{CO}(2-1)$ & $36.2 \pm 4.5$ & $-84.3 \pm 6.4$ & $124.3 \pm 15.9$ & $4.7 \pm 0.5$ & & \\
\hline East & {$[27,3]$} & $\mathrm{CO}(2-1)$ & $13.4 \pm 1.0$ & $-71.4 \pm 3.7$ & $127.5 \pm 9.9$ & $1.8 \pm 0.1$ & & 0.6 \\
\hline Test & {$[45,33]$} & CO10 & $\leq 3 \times 1.6$ & - & - & - & - & \\
\hline Test & {$[45,33]$} & $\mathrm{CO} 21$ & $\leq 3 \times 5.9$ & - & - & - & & \\
\hline
\end{tabular}

* Estimated without the HERA data. Note that the $\mathrm{CO}(1-0)$ could be half the value in the table here, see spectra on Fig. 2.

Table 2. Results of the observations.

\begin{tabular}{|c|c|c|c|c|c|c|c|c|}
\hline Position & $\begin{array}{l}\text { Offsets } \\
{\left[{ }^{\prime \prime} \times{ }^{\prime \prime}\right]}\end{array}$ & Line & $\begin{array}{c}T_{\mathrm{mb}} \\
{[\mathrm{mK}]} \\
\end{array}$ & $\begin{array}{l}\text { Velocity } \\
{\left[\mathrm{km} \mathrm{s}^{-1}\right]} \\
\end{array}$ & $\begin{array}{c}\text { Width } \\
{\left[\mathrm{km} \mathrm{s}^{-1}\right]}\end{array}$ & $\begin{array}{c}I_{\mathrm{CO}} \\
{\left[\mathrm{K} \mathrm{km} \mathrm{s}^{-1}\right]}\end{array}$ & $\begin{array}{c}M_{\mathrm{gas}} \\
{\left[10^{8} M_{\odot}\right]}\end{array}$ & $\overline{T_{21} / T_{10}}$ \\
\hline centre1 & {$[0,0]$} & $\mathrm{CO}(1-0)$ & $15.2 \pm 4.2$ & $-70.2 \pm 22.8$ & $333 \pm 69.1$ & $5.4 \pm 0.8$ & 12.6 & \\
\hline centre1 & {$[0,0]$} & $\mathrm{CO}(2-1)$ & $76 \pm 4.6$ & $-25 \pm 4.8$ & $301 \pm 11.5$ & $24.3 \pm 0.8$ & & \\
\hline centre1 & {$[0,0]$} & $\mathrm{CO}(2-1)$ & $26.7 \pm 1.6$ & $-41.2 \pm 4.5$ & $277.9 \pm 10.9$ & $7.9 \pm 0.26$ & & 1.7 \\
\hline centre2 & {$[-11,3]$} & $\mathrm{CO}(1-0)$ & $26.7 \pm 5.6$ & $-74.8 \pm 16.4$ & $238.6 \pm 41.3$ & $6.8 \pm 0.9$ & 15.8 & \\
\hline centre2 & {$[-11,3]$} & $\mathrm{CO}(2-1)$ & $83.7 \pm 17.2$ & $-99.7 \pm 14.8$ & $231.7 \pm 34.6$ & $20.6 \pm 2.6$ & & \\
\hline centre2 & {$[-11,3]$} & $\mathrm{CO}(2-1)$ & $31.1 \pm 1.2$ & $-63.8 \pm 2.9$ & $257.6 \pm 6.7$ & $8.5 \pm 0.2$ & & 1.2 \\
\hline centre3 & {$[11,-3]$} & $\mathrm{CO}(1-0)$ & $10.3 \pm 5.1$ & $-60.4 \pm 81.2$ & $340.3 \pm 216.2$ & $3.7 \pm 1.4$ & 8.7 & \\
\hline centre3 & {$[11,-3]$} & $\mathrm{CO}(2-1)$ & $29.7 \pm 10.2$ & $9.4 \pm 29.1$ & $340.2 \pm 69.2$ & $10.7 \pm 1.9$ & & \\
\hline centre3 & {$[11,-3]$} & $\mathrm{CO}(2-1)$ & $16 \pm 1.3$ & $-45.1 \pm 5.9$ & $248.4 \pm 15.4$ & $4.2 \pm 0.2$ & & 1.6 \\
\hline centre4 & {$[3,11]$} & $\mathrm{CO}(1-0)$ & $26.4 \pm 8.4$ & $-36.9 \pm 13.4$ & $83.1 \pm 29.6$ & $2.3 \pm 0.8$ & 5.4 & \\
\hline centre4 & {$[3,11]$} & $\mathrm{CO}(2-1)$ & $18.7 \pm 12.2$ & $-61.5 \pm 60.7$ & $320.4 \pm 97.8$ & $6.4 \pm 2.0$ & & \\
\hline centre4 & {$[3,11]$} & $\mathrm{CO}(2-1)$ & $17 \pm 1.1$ & $-44.7 \pm 4.9$ & $271.2 \pm 12.1$ & $4.9 \pm 0.2$ & & 0.6 \\
\hline centre5 & {$[-8,14]$} & $\mathrm{CO}(1-0)$ & $22.9 \pm 7.6$ & $-74.5 \pm 20.4$ & $167.5 \pm 43.3$ & $4.1 \pm 1.0$ & 9.5 & \\
\hline centre5 & {$[-8,14]$} & $\mathrm{CO}(2-1)$ & $33.2 \pm 10.1$ & $-76 \pm 21.6$ & $246 \pm 50$ & $8.7 \pm 1.5$ & & \\
\hline centre5 & {$[-8,14]$} & $\mathrm{CO}(2-1)$ & $23.8 \pm 0.6$ & $-61.3 \pm 1.7$ & $248.2 \pm 4.2$ & $6.3 \pm 0.1$ & & 1.0 \\
\hline centre6 & {$[-21,6]$} & $\mathrm{CO}(1-0)$ & $23.4 \pm 4.3$ & $-87.4 \pm 11.5$ & $191.3 \pm 31.9$ & $4.7 \pm 0.6$ & 11.1 & \\
\hline centre6 & {$[-21,6]$} & $\mathrm{CO}(2-1)$ & $-14.8 \pm 12.2$ & $165.9 \pm 68$ & $247.3 \pm 162.8$ & $-3.9 \pm 2.0$ & & \\
\hline centre6 & {$[-21,6]$} & $\mathrm{CO}(2-1)$ & $24 \pm 1.1$ & $-65.4 \pm 3.1$ & $246.7 \pm 7.1$ & $6.3 \pm 0.2$ & & 1 \\
\hline centre7 & {$[-3,-11]$} & $\mathrm{CO}(1-0)$ & $10 \pm 8.2$ & $-34.9 \pm 79.2$ & $441.4 \pm 209.8$ & $4.7 \pm 1.8$ & 11 & \\
\hline centre7 & {$[-3,-11]$} & $\mathrm{CO}(2-1)$ & $32.4 \pm 7.6$ & $-164.8 \pm 12.8$ & $117 \pm 39.8$ & $4.0 \pm 1.0$ & & \\
\hline centre7 & {$[-3,-11]$} & $\mathrm{CO}(2-1)$ & $15.5 \pm 1.1$ & $-32.7 \pm 5.6$ & $277.4 \pm 13.8$ & $4.5 \pm 0.2$ & & 1.6 \\
\hline centre8 & {$[-18,17]$} & $\mathrm{CO}(1-0)$ & $14.1 \pm 2.2$ & $-82.9 \pm 8.5$ & $142.8 \pm 16.2$ & $2.1 \pm 0.2$ & 5 & \\
\hline centre8 & {$[-18,17]$} & $\mathrm{CO}(2-1)$ & $-28.2 \pm 6.2$ & $121.1 \pm 14.5$ & $47.6 \pm 28.2$ & $-1.4 \pm 0.5$ & & \\
\hline centre8 & {$[-18,17]$} & $\mathrm{CO}(2-1)$ & $21.1 \pm 1.1$ & $-56.1 \pm 3.5$ & $241.4 \pm 8.2$ & $5.4 \pm 0.2$ & & 1.5 \\
\hline centre9 & {$[-24,-5]$} & $\mathrm{CO}(1-0)$ & $16.2 \pm 5.6$ & $-149.5 \pm 16.3$ & $114.3 \pm 36.4$ & $2.0 \pm 0.6$ & 4.6 & \\
\hline centre9 & {$[-24,-5]$} & $\mathrm{CO}(2-1)$ & $-48.9 \pm 18.4$ & $55.2 \pm 15.4$ & $63.7 \pm 33.1$ & $-3.3 \pm 1.4$ & & \\
\hline centre9 & {$[-24,-5]$} & $\mathrm{CO}(2-1)$ & $12.4 \pm 1.0$ & $-64.2 \pm 5.9$ & $275.1 \pm 14.3$ & $3.6 \pm 0.2$ & & 0.8 \\
\hline
\end{tabular}

similar. The $\mathrm{CO}(2-1)$ line fluxes, when not convolved with the $\mathrm{CO}(1-0)$ beam, are 4 times larger than the $\mathrm{CO}(1-0)$ fluxes. So within the uncertainties, this is in agreement with an optically thick cold gas. After convolution, all the ratio are close to one. Some regions have a ratio greater than one, but as mentioned before, the $\mathrm{CO}(1-0)$ lines are affected by baseline ripples close to $3 \mathrm{C} 84$. The main beam brightness temperatures in $\mathrm{CO}(1-0)$ are thus very likely under-estimated in this region.

\subsection{Detection of $\mathrm{HCN}(3-2)$}

We searched for $\mathrm{HCN}(1-0), \mathrm{HCN}(2-1)$ and $\mathrm{HCN}(3-2)$ emission lines in the centre of NGC 1275. The observations at low frequency were corrupted by strong baseline ripples due to the strong continuum source $3 \mathrm{C}$ 84. So we focus on the $\mathrm{HCN}(3-2)$ observations at $261 \mathrm{GHz}$, observed in average weather conditions. The spectrum presented in Fig 5 is the average of data taken during a first run with the two receivers detecting two 


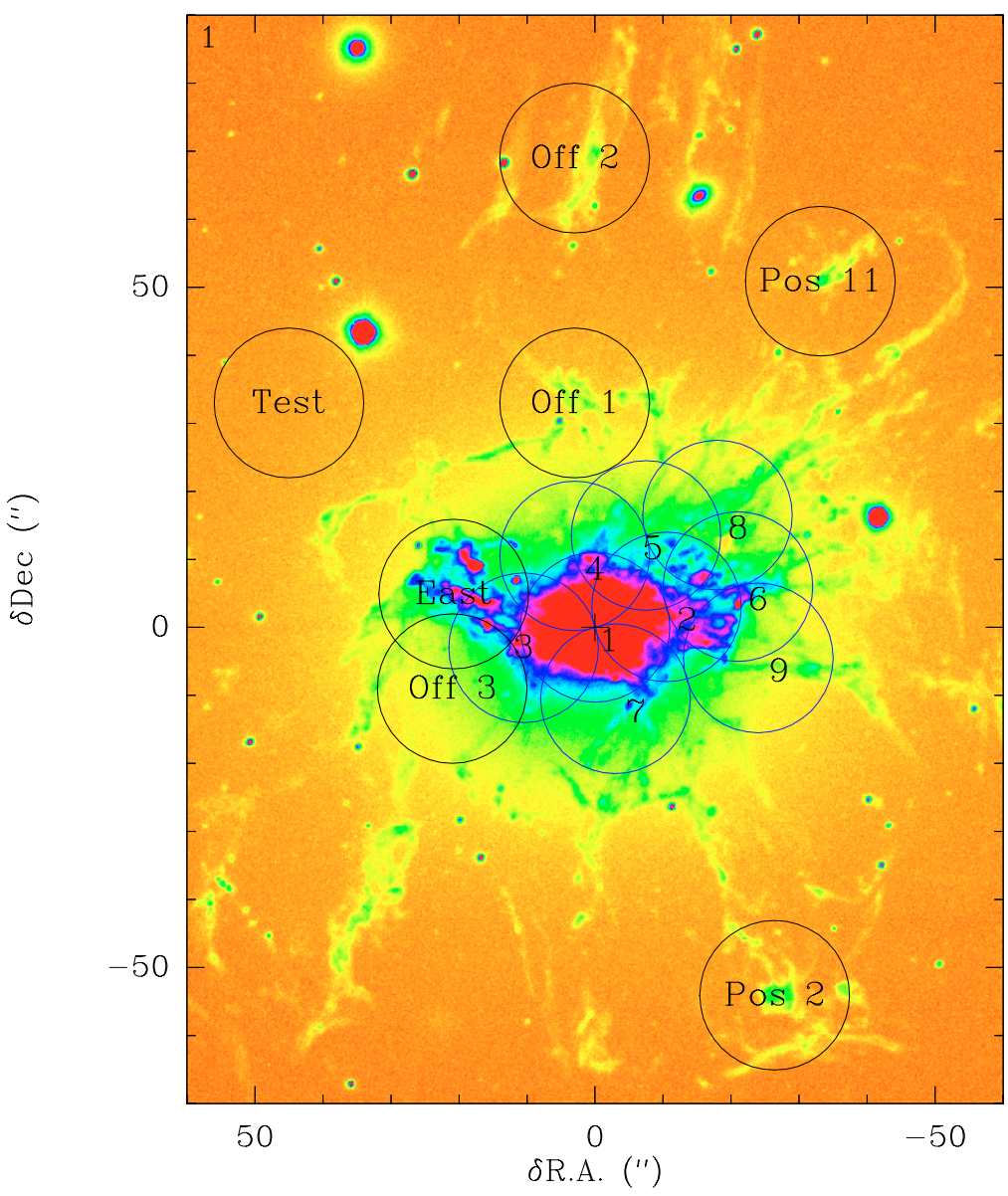

Fig. 1. $\mathrm{H} \alpha$ image of the filament system in NGC 1275 (Conselice et al. 2001). Overlaid are the regions re-observed with the $30 \mathrm{~m}$ telescope. The circles represent the beam size at $3 \mathrm{~mm}$. polarisations (A230, B230) and during a second run with the two other receivers C270 and D270. The signal appears marginally in both data sets and even better in the averaged spectrum. So we claim a detection of $\mathrm{HCN}(3-2)$. The $\mathrm{S} / \mathrm{N}$ ratio on the integrated line is 6.

The HCN molecule has a large dipole moment that requires $n\left(\mathrm{H}_{2}\right)>10^{4} \mathrm{~cm}^{-3}$ for significant excitation. This tracer of dense gas regions is thus considered as an indicator of star formation activity (Gao \& Solomon, 2004). We computed the apparent $\mathrm{CO}$ and $\mathrm{HCN}$ luminosities (using Eq. (1) from Downes et al. 1999) from the integrated line flux measured in $\mathrm{CO}(1-0)$ and $\mathrm{HCN}(3-2)$. We assumed a $\mathrm{HCN}(3-2) / \mathrm{HCN}(1-0)$ ratio close to one. At densities higher than a few $10^{4} \mathrm{~cm}^{-3}$ for a temperature higher that $40-50 \mathrm{~K}$, radiative transfer models predict a ratio larger than $\sim 0.25$. We found $L_{\mathrm{CO}}^{\prime}=5 \times 10^{8} \mathrm{~K} \mathrm{~km} \mathrm{~s}^{-1} \mathrm{pc}^{2}$ and $L_{\mathrm{HCN}}^{\prime}=5 \times 10^{7} \mathrm{~K} \mathrm{~km} \mathrm{~s}^{-1} \mathrm{pc}^{2}$. When compared to the $L_{\mathrm{FIR}}-L_{\mathrm{CO}}^{\prime}$ and $L_{\mathrm{FIR}}-L_{\mathrm{HCN}}^{\prime}$ relations as described by Solomon et al. (1992, 1997), our measurements fall in the normal spiral galaxies range and independently predict an $L_{\text {FIR }}$ of $2 \times 10^{10} L_{\odot}$. Lester et al. (1995) estimated the thermal FIR emission to be $10^{11} L_{\odot}$, with the approximation that only $20 \%$ of the total luminosity was nonthermal. This is an order of magnitude higher than the $L_{\mathrm{FIR}}$ predicted here. So either the contribution of the synchrotron emission is larger than expected or there is an excess of FIR emission in NGC 1275 compared to the well-known $L_{\mathrm{FIR}}-\mathrm{L}_{\mathrm{HCN}}^{\prime}$ relation for star forming galaxies.

\subsection{Molecular gas from the high velocity component}

NGC 1275 is surrounded by some star formation regions, with young star clusters, that could be formed in a merger but also in the cooling flow (see S06). Some are associated with the High Velocity System (HVS) at $8200 \mathrm{~km} \mathrm{~s}^{-1}$, which appears in absorption in front of the optical emission of NGC 1275 (Gillmon et al. 2004). No stellar component is detected, even in the nearinfrared, corresponding to the HVS, which could be a gas-rich disrupted dwarf, or tidal debris, as proposed by Hu et al. (1983).

We searched for CO emission at $8280 \mathrm{~km} \mathrm{~s}^{-1}$ on one position in the HVS (centred on 3C 84's position). The results are shown in Fig. 6. There is no clear detection but a hint of $\mathrm{CO}(1-$ $0)$ emission. The line intensity we derive is up to a maximum of the order of $0.4 \mathrm{~K} \mathrm{~km} \mathrm{~s}^{-1}$ which corresponds to $\leq 10^{8} M_{\odot}$ of cold gas. So even if interacting with the ambient medium closely surrounding NGC 1275, the HVS contains a very small amount of cold gas. This mass is comparable or less than what is found in the cold filaments around the cD galaxy. Therefore, if the HVS merges with the cD galaxy, it will not deposit a significant amount of cool gas.

\section{Discussion}

\section{1. $\mathrm{CO}(2-1) / \mathrm{CO}(1-0)$ line ratios}

We have computed the $\mathrm{CO}(2-1) / \mathrm{CO}(1-0)$ line ratios. The data at $1.3 \mathrm{~mm}$ were combined with the HERA data described in S06. We then convolved the $\mathrm{CO}(2-1)$ data by the $\mathrm{CO}(1-0)$ beam size in order to make a meaningful comparison. We list, in Table 1 , the $\mathrm{CO}(2-1) / \mathrm{CO}(1-0)$ line ratios of the different regions observed. If we compare the main beam temperature in the $\mathrm{CO}(2-1)$ spectra with and without the $3 \mathrm{~mm}$ beam shape convolution (central and right hand side column of Fig. 2), we can see that we have lost some signal by diluting the $1.3 \mathrm{~mm}$ emission 

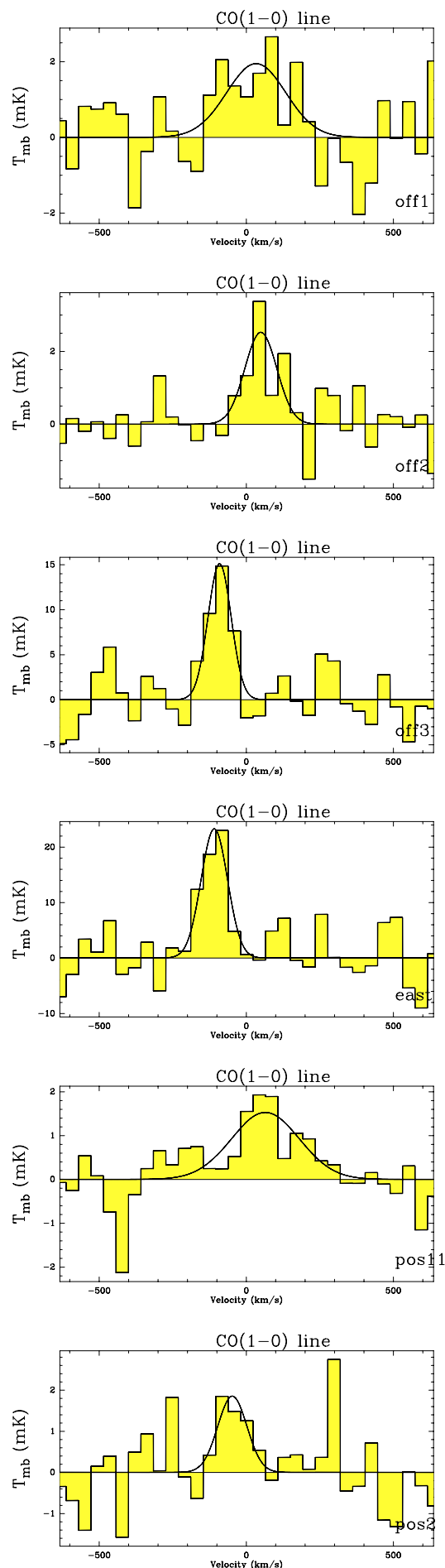
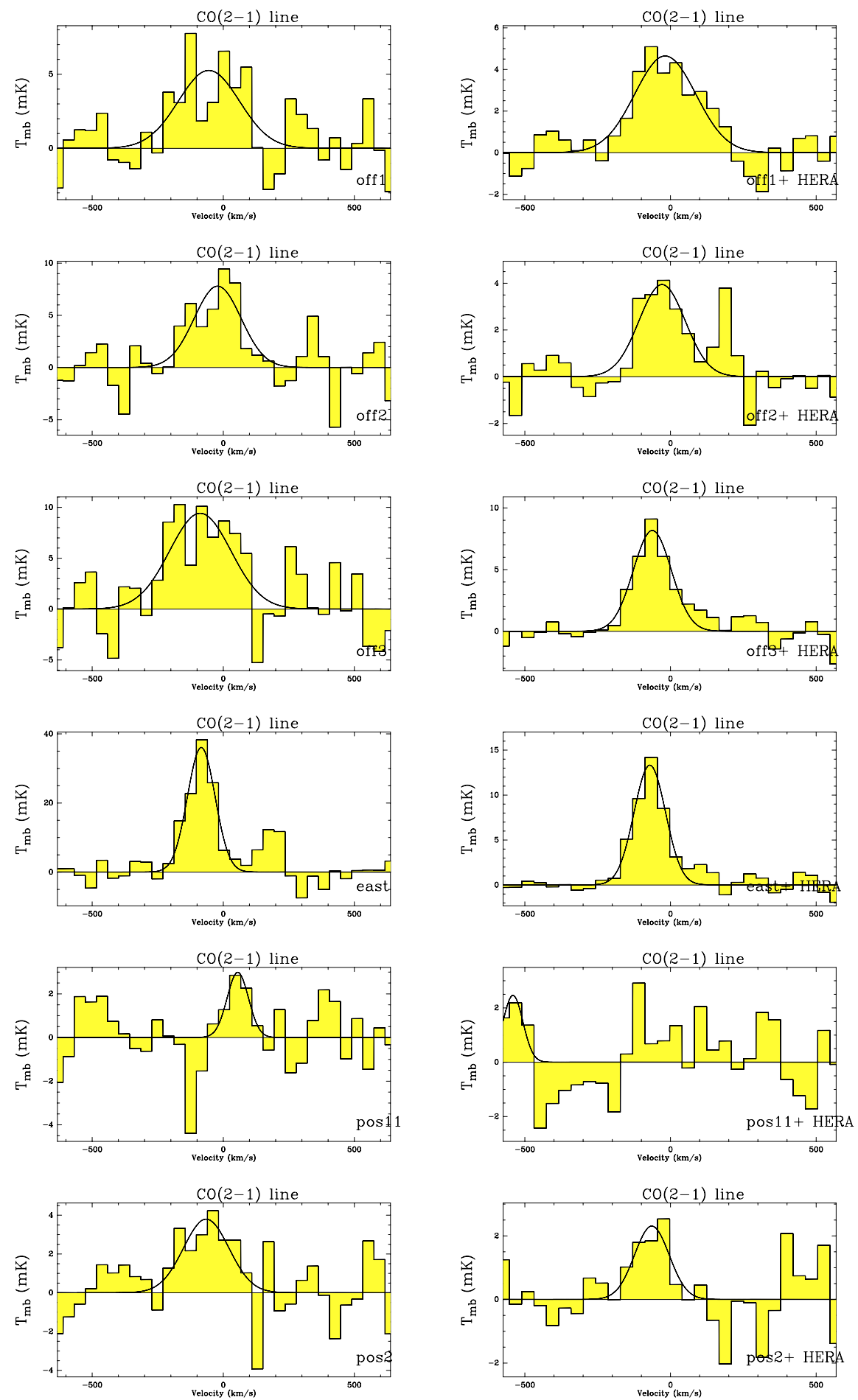

Fig. 2. $\mathrm{CO}(1-0)$ and $\mathrm{CO}(2-1)$ spectra obtained at all the positions observed as indicated at lower right in each diagram. The channel width is $42 \mathrm{~km} \mathrm{~s}^{-1}$. On the left hand side are the $\mathrm{CO}(1-0)$ lines detected with the a100 and b100 receivers. In the middle are the results obtained for the $\mathrm{CO}(2-1)$ line with the $\mathrm{A} 230$ and $\mathrm{B} 230$ receivers. On the right hand side are the $\mathrm{CO}(2-1)$ lines computed with both $\mathrm{A} 230$ and $\mathrm{B} 230$ merged with previous HERA data and smoothed to the $3 \mathrm{~mm}$ beam size.

with a larger beam size. This means that the molecular gas emission is very likely to come from a region that is smaller than the $1.3 \mathrm{~mm}$ beam size $\left(11^{\prime \prime}\right)$.

At Pos 11, the tentative detection of $\mathrm{CO}(2-1)$ disappears when convolved to the $\mathrm{CO}(1-0)$ beam. Regions Off 2 , Pos 2 , Pos 11 and East show lower main beam temperatures at $1.3 \mathrm{~mm}$ when we applied the $3 \mathrm{~mm}$ beam convolution. Therefore, the emission from these positions probably comes from regions with an angular size less than $22^{\prime \prime}$ (the beam size at $3 \mathrm{~mm}$ ). Regions Off 1 and Off 3 do not show lower main beam temperatures when convolved with the $3 \mathrm{~mm}$ beam and are therefore probably extended by at least $22^{\prime \prime}$. We have recently confirmed this 

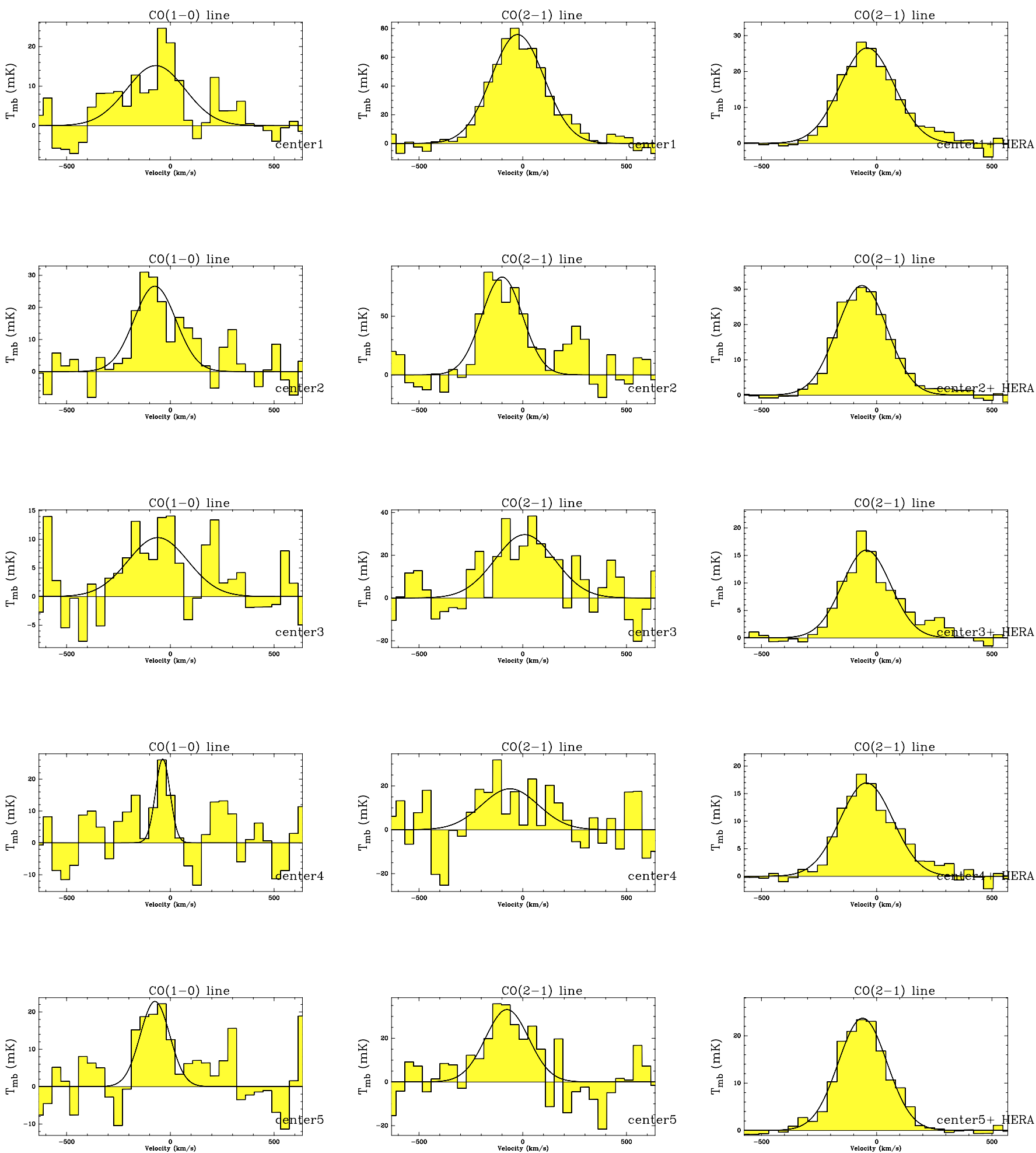

Fig. 3. $\mathrm{CO}(1-0)$ and $\mathrm{CO}(2-1)$ spectra obtained at all the positions near the centre of the galaxy as labeled in the lower right of each diagram. The channel width is $42 \mathrm{~km} \mathrm{~s}^{-1}$, see Table 2 .

last result by mapping the eastern filament with the Plateau de Bure interferometer. This shows that the molecular gas lies in thin $\left(\leq 2^{\prime \prime}\right)$ and elongated structures, exactly coincident with the H $\alpha$ filaments (Salomé et al. 2008).

After convolution to the same beam size, we find the $\mathrm{CO}(2-1) / \mathrm{CO}(1-0)$ line brightness temperature ratio is close to one in regions East, Off3, Pos 2 and close to $3 \mathrm{C} 84$. Such a ratio indicates optically thick $\mathrm{CO}(1-0)$ and $\mathrm{CO}(2-1)$ emission lines (Eckart et al. 1988). For regions farther out from the galaxy centre like Off2, the ratio is slightly larger than one. However, we sometimes find the line widths smaller in $\mathrm{CO}(1-0)$ than in $\mathrm{CO}(2-1)$. This means that the apparently high ratio could actually be closer to one if the line widths were constrained to be the same. Note that the Off 1 emission-line ratio is about 2.5 while 

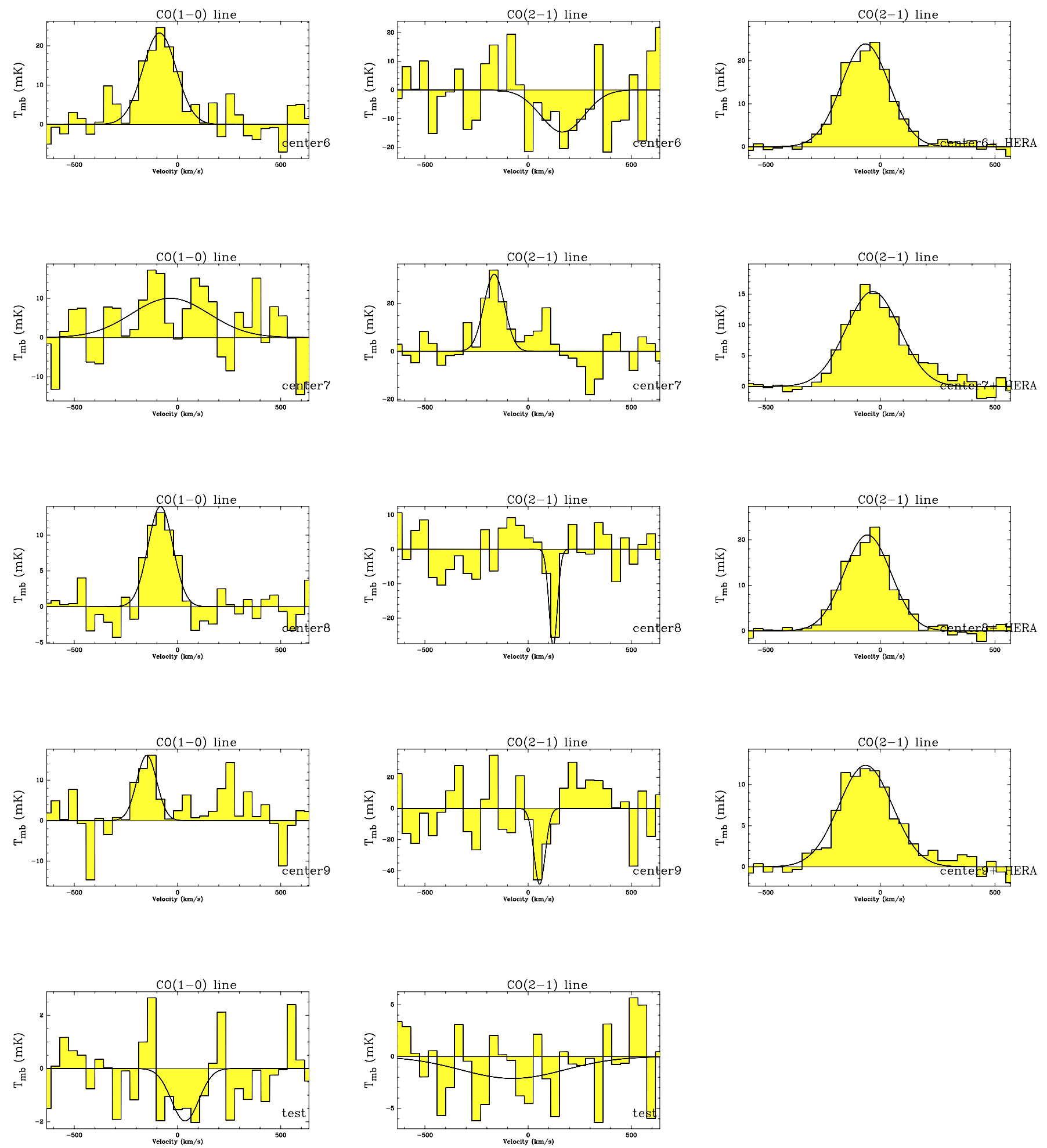

Fig. 4. $\mathrm{CO}(1-0)$ and $\mathrm{CO}(2-1)$ spectra obtained at all the positions near the centre of the galaxy as labeled in the lower right of each diagram. The channel width is $42 \mathrm{~km} \mathrm{~s}^{-1}$, see Table 2 .

the line shapes are the same at $1.3 \mathrm{~mm}$ and $3 \mathrm{~mm}$. So we see hints of higher $\mathrm{CO}(2-1) / \mathrm{CO}(1-0)$ ratios at larger radius from the galaxy centre. This particular high ratio (larger than one) indicates optically thin emission (Goldsmith et al. 1999).

Closer to the centre, the strong $3 \mathrm{C} 84$ source produced baseline ripples that affected the $3 \mathrm{~mm}$ line shapes. However, we estimated $\mathrm{CO}(2-1) / \mathrm{CO}(1-0)$ line ratios close to one, in agreement with an optically thick cold gas (see Table 2).

\subsection{Molecular gas reservoir and star formation}

The scenario for the origin of the molecular gas we detect is that the cold gas accumulating towards the centre of the cluster gets dragged out with the bubbles and compressed in curved shocks, or flows back down around the borders of cavities. Feedback from the AGN not only heats the gas (negative feedback), but also compresses it in thin layers, that favors cooling and 


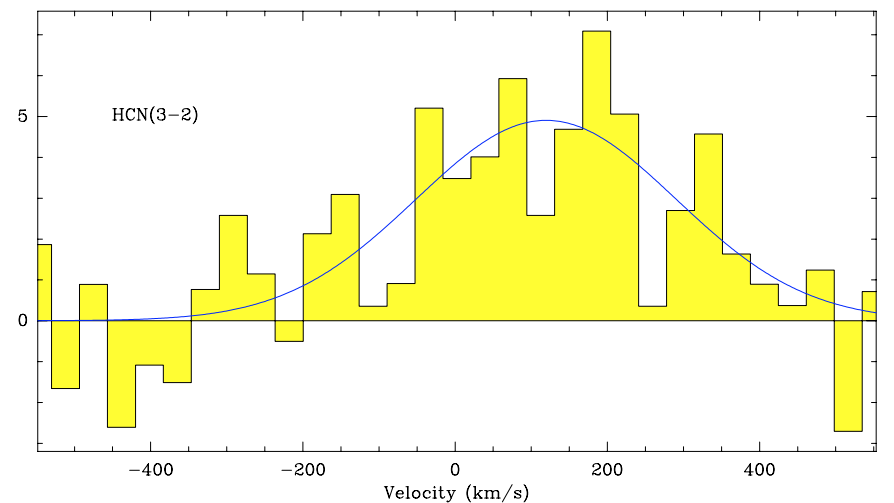

Fig. 5. HCN(3-2) emission lines in $T_{\mathrm{mb}}(\mathrm{mK})$, observed with the $30 \mathrm{~m}$ telescope $(290 \mathrm{~min})$. A gaussian fit gives $I_{\mathrm{HCN}} 2.1 \mathrm{~K} \mathrm{~km} \mathrm{~s}^{-1}$ with a peak temperature of $5 \mathrm{mK}$, a velocity of $120 \mathrm{~km} \mathrm{~s}^{-1}$ and a linewidth of $400 \mathrm{~km} \mathrm{~s}^{-1}$. The rms is $1.6 \mathrm{mK}$ with a mean $T_{\text {sys }}$ of $445 \mathrm{~K}$. The channel width is $36 \mathrm{~km} \mathrm{~s}^{-1}$.

formation of molecular filaments (positive feedback) at a distance greater than $10 \mathrm{kpc}$ (Revaz et al. 2008).

We expect no significant amounts of dust in the filaments, due to high sputtering rates. The question is then whether, in these conditions, there is still sufficient dust (which acts as a catalyst) to form molecules quickly. It may be that some dust from the ISM of NGC 1275 has been captured inside the uplifted cooler gas and this is sufficient to catalyse the formation of molecules. Dust could also form in interstellar shocks, if present. The presence of dust grains at large radii would then help to form molecules in the outer filaments. A small quantity of dust grains is sufficient. We have proposed further observational investigations in order to constrain the amount of dust inside the filaments of NGC 1275.

For the assumed standard $\mathrm{CO} / \mathrm{H}_{2}$ conversion factor, the amount of molecular gas detected in the different regions observed varies from $7 \times 10^{7} M_{\odot}$ to $8 \times 10^{8} M_{\odot}$, which when added together gives a total mass of $1.3 \times 10^{9} M_{\odot}$. These filaments therefore contain large quantities of cold molecular gas. The regions are also conspicuous in the emission lines of $\mathrm{H} \alpha$ and [NII] but the optical line ratios are incompatible with young stars as the source of excitation as might be expected if stars were forming from the molecular gas. The excitation of these lines is not coming from the AGN either, since it does not decrease with distance from the nucleus of NGC 1275 (Hatch et al. 2006). The filaments are emitting extensively in UV and optical lines, much more than in X-rays, and a possible explanation for this is through heating by shocks at the interface between the relativistic jet plasma and the entrained gas at the exterior of the bubbles.

One of the most CO-rich regions is the eastern extension, which contains an unresolved $(<1 \mathrm{kpc})$ young star cluster (Shields et al. 1990). The molecular gas mass of this region is greatly in excess of the mass of the star cluster and confirms a low star formation efficiency within the filaments (see also Salomé et al. 2008, for more details).

\section{Conclusions}

New $\mathrm{CO}(1-0)$ and $\mathrm{CO}(2-1)$ observations towards selected optical filaments around NGC 1275 confirm the presence of CO associated with the $\mathrm{H} \alpha$ filaments. The gas kinematics are the same in $\mathrm{CO}$ and as in $\mathrm{H} \alpha$. We detect for the first time, a large amount of cold gas inside the outer filaments (a few $10^{8} M_{\odot}$ ).
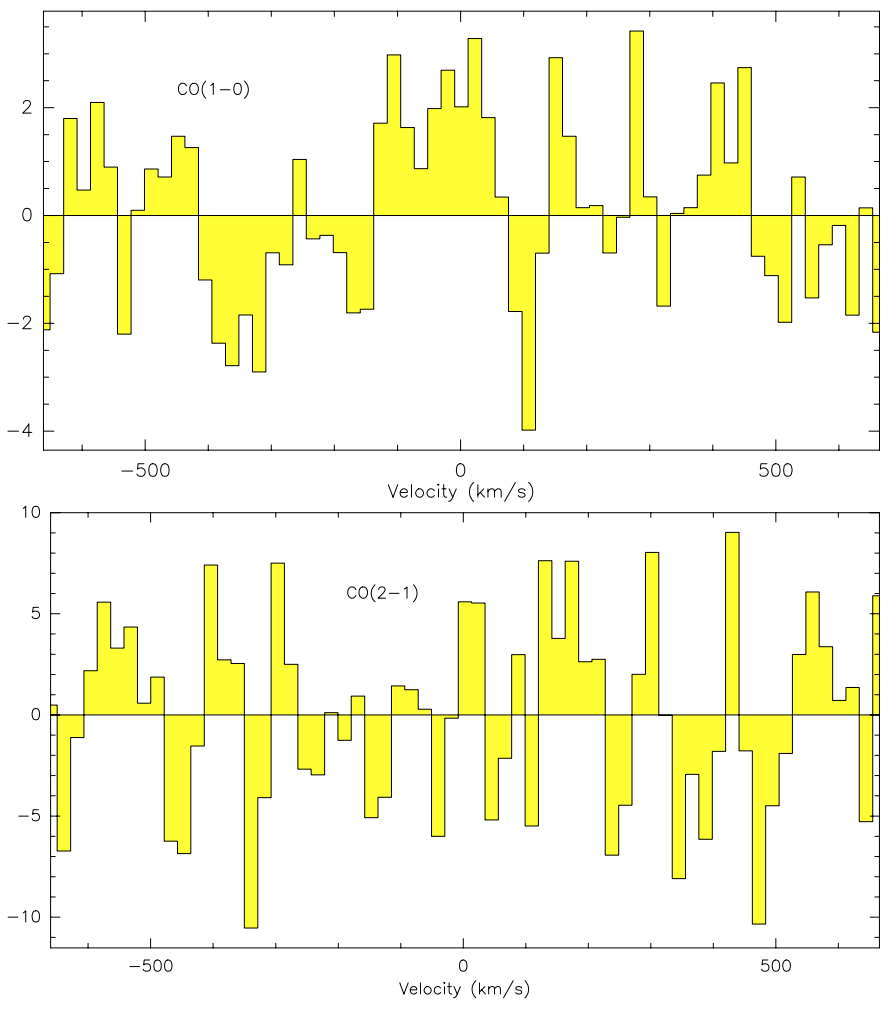

Fig. 6. Observations at $112.175 \mathrm{GHz}$ (top) and $224.346 \mathrm{GHz}$ (bottom), the $\mathrm{CO}(1-0)$ and $\mathrm{CO}(2-1)$ frequencies expected in the HVS restframe at $8280 \mathrm{~km} \mathrm{~s}^{-1}$. No $\mathrm{CO}(2-1)$ is detected. There is a hint of detection in $\mathrm{CO}(1-0)$, with $I_{\mathrm{CO}}=0.4 \pm 0.1 \mathrm{~K} . \mathrm{km} \mathrm{s}^{-1}$. This would give a mass of about $1 \times 10^{8} M_{\odot}$. The channel width is $21 \mathrm{~km} \mathrm{~s}^{-1}$.

The excitation of the gas, derived from the comparison of $\mathrm{CO}(2-1)$ and $\mathrm{CO}(1-0)$ convolved to the same spatial resolution, indicates a range of densities are present. Optically thick emission is present close to the galaxy centre, whilst further out the emission could be optically thin.

We report a detection of $\mathrm{HCN}(3-2)$ emission in the centre, indicating high density gas. The association of the molecular gas with the $\mathrm{H} \alpha$ filaments is consistent with the scenario in which cooling of the gas is taking place at large radii. This could happen in compressed hot gas at the border of the X-ray cavities. A detailed interpretation awaits specific simulations taking into account the cold, warm and hot gas phases.

Acknowledgements. IRAM is supported by INSU/CNRS (France), MPG (Germany) and IGN (Spain). R.M.J. acknowledges support by STFC and the Royal Society. We thank D. Downes for helpful comments and discussions.

\section{References}

Binney, J., \& Tabor, G. 1995, MNRAS, 276, 663

Braine, J., Duc, P.A., Lisenfeld, U., et al. 2001, A\&A 378, 51

Brüggen, M ., \& Kaiser, C. R. 2002, Nature, 418, 301

Carlson, M. N., Holtzman, J. A., Watson, A. M., et al. 1998, AJ, 115, 1778

Cattaneo, A., \& Teyssier, R. 2007, 376, 1547

Conselice, C. J., Gallagher, J. S., \& Wise, R. F. G. 2001, AJ, 122, 2281

Crawford, C. S., Allen, S. W., Ebeling, H., et al. 1999, MNRAS, 306, 857

Downes, D., Neri, R., Wiklind, T., et al. 1999, AJ, 513, L4

Eckart, A., Downes, D., Genzel, R., et al. 1990, 348, 434

Edge, A. C. 2001, MNRAS, 328, 762

Edwards, L. O. V., Hudson, M. J., Balogh, M. L., \& Smith, R. J. 2007, MNRAS, 319,100 
Fabian, A. C., Sanders, J. S., Allen, S. W., et al. 2003, MNRAS, 344, L43

Gao, Y. \& Solomon, P. M. 2004, ApJ, 606, 271

Gillmon, K., Sanders, J. S., \& Fabian, A. C. 2004, MNRAS, 348, 159

Goldsmith, P. F., \& Langer, W. D. 1999, 517, 209

Gonzalez-Martin, O., Fabian, A. C., \& Sanders, J. S. 2006, MNRAS, 367, 1132

Hatch, N. A., Crawford, C. S., Fabian, A. C., \& Johnstone, R. M. J 2005, MNRAS, 358, 765

Hatch, N. A., Craxford, C. S., Johnstone, R. M., \& Fabian A. C. 2006, MNRAS, 367,433

Hibbard, J. E., \& van Gorkom, J. H. 1996, AJ, 111, 655

Higdon, S. J., Higdon, J. L., \& Marshall, J. 2006, ApJ, 640, 768

Hu, E. M., Cowie, L. L., Kaaret, P., et al. 1983, ApJ, 275, L27

Inoue, M. Y., Kameno, S., Kawabe, R., et al. 1996, AJ, 111, 1852

Lester, D. F., Zink, E. C., Doppmann, G. W., et al. 1995, ApJ, 439, 185

McNamara, B. R., O’Connell, R. W., \& Sarazin, C. L., 1996, AJ, 112, 91
Omma, H., \& Binney, J. 2004, MNRAS, 350L, 13

Revaz Y., Combes, F., \& Salomé, P. 2008, A\&A

Salomé, P. \& Combes, F. 2003, A\&A, 412, 657

Salomé, P. \& Combes, F. 2004, A\&A, 415, L1

Salomé, P., Combes, F., Edge, A. C., et al. 2006, 454, 437

Salomé, P., Revaz, Y., Combes, F., et al. 2008, A\&A, in press [arXiv: 0804.0694]

Shields, J. C., \& Filippenko, A. V. 1990, ApJ, 353, L6

Sijacki, D., \& Springel, V. 2006, MNRAS 366, 397

Solomon, P. M., Downes, D., \& Radford, S. J. E. 1992, ApJ, 387, L55

Solomon, P. M., Downes, D., Radford, S. J. E., \& Barrett, J. W. 1997, ApJ, 478, 144

Wilman, R. J., Edge, A. C., \& Johnstone, R. M. 2005, MNRAS, 359, 755

Xu, C. K., Lu, N., Condon, J. J. et al. 2003, ApJ, 595, 665 